\title{
Can admission lipoprotein-associated phospholipase A2 predict the symptomatic cerebral vasospasm following aneurysmal subarachnoid hemorrhage?
}

Chen-Yu Ding ${ }^{\dagger}$, Fang-Yu Wang ${ }^{\dagger}$, Han-Pei Cai, Xiao-Yong Chen, Shu-Fa Zheng, Liang-Hong Yu, Yuan-Xiang Lin, Zhang-Ya Lin and De-Zhi Kang*

\begin{abstract}
Background: Inflammation has been believed to be related to the development of cerebral vasospasm following aneurysmal subarachnoid hemorrhage (aSAH). A potential biomarker for vascular inflammation that is well recognized is the lipoprotein-associated phospholipase A2 (Lp-PLA2). However, whether Lp-PLA2 can predict the occurrence of symptomatic cerebral vasospasm (SCV) in aSAH patients is still unknown. Thus, this study aimed to assess the value of Lp-PLA2 for predicting SCV in patients with aSAH.
\end{abstract}

Methods: Between March 2017 and April 2018, we evaluated 128 consecutive aSAH patients who were admitted in the First Affiliated Hospital of Fujian Medical University. Their Lp-PLA2 level was obtained within $24 \mathrm{~h}$ of the initial bleeding. Factors might be related to SCV were analyzed.

Results: Compared to patients without SCV, those with SCV $(9.4 \%, 12 / 128)$ had significantly higher Lp-PLA2 level. Multivariate logistic analysis revealed that worse modified Fisher grade $(\mathrm{OR}=10.08,95 \% \mathrm{Cl}=2.04-49.86, P=0.005)$ and higher $\mathrm{Lp}$-PLA2 level $(\mathrm{OR}=6.66,95 \% \mathrm{Cl}=1.33-3.30, P=0.021)$ were significantly associated with SCV, even after adjustment for confounders. Based on the best threshold, LP-PLA2 had a sensitivity of $83.3 \%$ and a specificity of $51.7 \%$ for predicting SCV, as shown by the receiver operating characteristic curve analysis. In the poor World Federation of Neurosurgical Societies grade patient sub-group, patients with Lp-PLA2 > $200 \mu \mathrm{g} / \mathrm{L}$ had significantly higher SCV rate than that of patients having Lp-PLA2 $\leq 200 \mu \mathrm{g} / \mathrm{L}$.

Conclusion: The admission LP-PLA2 level might be a helpful predictor for SCV in aSAH.

Keywords: Aneurysmal subarachnoid hemorrhage, Vasospasm, Biological markers

\footnotetext{
* Correspondence: kdz99999@sina.com

${ }^{\dagger}$ Chen-Yu Ding and Fang-Yu Wang are co-first authors.

Department of Neurosurgery, The First Affiliated Hospital of Fujian Medical

University, Fuzhou, Fujian, People's Republic of China
} 


\section{Background}

One of the most frequent and worst complications of aneurysmal subarachnoid hemorrhage (aSAH) is cerebral vasospasm $(\mathrm{CV})$, which leads to the highest mortality in aSAH patients [1]. CV of aSAH could lead to cerebral ischemia or infraction, which worsens patient condition. Further development of ischemia is possible if the condition is not controlled in time, and it could result in delayed ischemic neurological deficits that threat the life of patient $[2,3]$. Therefore, it is of clinical significance to understand factors associated with development of $\mathrm{CV}$, as they may help clinicians to identify individuals at risk and potentially prevent this complication.

Currently, clinical rating scales such as the World Federation of Neurosurgical Societies (WFNS) grade and modified Fisher grade, which are easy to use and have relatively high predictive power, are used for assessing the performance of neurological functions and predicting the occurrence of CV [4-6]. However, these clinical scales are not able to differentiate the physical condition of a patient when the changes are small. For example, it is possible for the Fisher scale to categorize all thick clots as grade III, therefore fails to differentiate increasing thickness of subarachnoid clots [7]. To overcome this disadvantage, a number of alternative grading schemes have been proposed. However, each scheme is associated with certain limitations [6-11]. Currently, there is still an unmet need for reliable biomarkers that could be used for more effective diagnosis and assessment of aSAH [12-14] in the clinical setting.

The onset of CV has a complicated mechanism involving many factors. Studies have shown that inflammatory response and inflammatory factors are critically involved in every step of the CV development cascade. Lipoproteinassociated phospholipase A2 (Lp-PLA2) is an available potential biomarker of inflammation in blood vessels [15-18]. It has been reported that in aSAH patients, elevated LpPLA2 level is related to angiographic cerebral vasospasm (ACV) [19]. However, whether Lp-PLA2 can predict the occurrence of symptomatic cerebral vasospasm (SCV) in aSAH patients is still unknown. Results from previous studies regarding predictors of $\mathrm{CV}$ after aSAH are inconsistent [20], which is mainly caused by using different definitions or diagnostic criteria for CV. For example, both ACV and SCV have been used to define CV $[1,21]$, but the associated morbidity and mortality of SCV could be higher than those of ACV in aSAH patients. Therefore, this study aimed to investigate the predictive value of Lp-PLA2 level for SCV following aSAH.

\section{Methods}

\section{Study population}

Based on a previous single-center prospective and observational cohort study [22], this study is also observational and prospective, but with additional patients enrolled. Enrollment included aSAH patients admitted to our department between March 2017 and April 2018, but only those consecutive patients who had their post-aSAH Lp-PLA2 level measured within the first $24 \mathrm{~h}$ were included for analysis.

The following inclusion criteria were used [22]: (1) admitted to the hospital within $24 \mathrm{~h}$ of SAH onset; (2) $\mathrm{SAH}$ was caused by intracranial aneurysm as confirmed via computed tomography angiography (CTA) or digital subtraction angiography (DSA); (3) aneurysm was treated, either with clipping or interventional method, within $48 \mathrm{~h}$ of admission; and (4) blood Lp-PLA2 level at admission was obtained. The following exclusion criteria were used: (1) patient had surgery or severe infection within the past month; (2) previous occurrence of $\mathrm{SAH}$ or had other neurological diseases such as ischemic stroke, hemorrhagic stroke, or severe head trauma; (3) previous use of antiplatelet and anticoagulant drugs or had a history of immunotherapy; and (4) other systemic diseases such as autoimmune disease, uremia, cirrhosis, cancer, chronic lung diseases, and chronic heart diseases including coronary artery diseases and myocardial infarction.

The study protocol was designed in accordance with guidelines outlined in the Declaration of Helsinki and approved by the Ethics Committee of the First Affiliated Hospital of Fujian Medical University (Fujian, China). Informed consent was obtained from the patients or their authorized legal representative if patients cannot sign the form themselves.

\section{Patient management}

The guidelines of American Heart Association and American Stroke Association were used for clinical management [23]. The guideline of Neurocritical Care Society was used for critical care management [24]. Since the day of hospitalization, all patients received nimodipine, either orally $(6 \times 60 \mathrm{mg} /$ day $)$ or intravenously $(2 \mathrm{mg} / \mathrm{h})$.

\section{Clinical and radiological variables}

Medical history, admission conditions, imaging data, prior treatment, and relevant clinical information were collected for every patient. The amount of subarachnoid blood presented on the admission CT image was semiquantified with the modified Fisher grade. The severity of aSAH was evaluated using the initial Hunt \& Hess scale, Glasgow coma scale (GCS), and WFNS grade. Each patient was assigned a grade, which was the average score given by two doctors. If a large deviation was seen, a third, more senior doctor will determine the final score. Good grade was defined as admission WFNS grade $<3$, and poor grade was defined as admission WFNS grade $\geq 3$. 
Lp-PLA2 level at admission was measured during the routine patient care procedure. After patient admission, venous blood was drawn and stored in anti-coagulation tube with ethylenediaminetetraacetic acid. The collected blood was centrifuged at room temperature at $1000 \mathrm{~g}$ for 20 min. The supernatant was collected and stored in $80{ }^{\circ} \mathrm{C}$ freezer until analysis. To measure the plasma LpPLA2 level, double-antibody sandwich enzyme-linked immunosorbent assay (ELISA) was performed in this study (Cloud-Clone Corp, Houston, TX, USA), following the protocol provided by the manufacturer. Detection limit of the kit was $0.286 \mu \mathrm{g} / \mathrm{L}$, coefficient of variation $(\mathrm{COV})$ within the assay was $<10 \%$, and intra-assay COV was $<12 \%$. Lp-PLA2 level $>200 \mu \mathrm{g} / \mathrm{L}$ indicated moderate vascular inflammation [25]. Good Lp-PLA2 level was defined as admission Lp-PLA2 level $<200 \mu \mathrm{g} / \mathrm{L}$, and poor level was defined as admission Lp-PLA2 level $\geq 200 \mu \mathrm{g} / \mathrm{L}$.

\section{Detection of cerebral vasospasm}

Detection of cerebral vasospasm using computed tomography angiography (CTA) (Acquilion ONE, Toshiba Medical Systems, Nasu, Japan) or 3.0 T three-dimensional time-of-flight (TOF) MRA (Magnetom Verio Tim, Siemens, Erlangen, Germany; or another system: Skyra; Siemens, Erlangen, Germany) was performed for all patients.

Two neurosurgeons, blinded to the patient's pre- and post-operative medical history, performed analysis using CTA/MRA. When a new infarction on diffusion-weighted imaging was not immediately visible after operation and clinical deterioration/neurological deficit attributable to vascular narrowing was found on MRA, CTA, or digital subtraction angiography (DSA), it was defined as SCV.

At pre-operation and the first day post-operation, CTA/MRA was performed, and again at every 3 to 7 days until day 30, based on the decision made by the neurosurgeon and neurocritical care physician. If $\mathrm{CV}$ was identified, daily transcranial Doppler sonography, CTA, or MRA was performed until CV was resolved. DSA was performed if CV was suspected and CTA/ MRA became unsuitable for analyzing the spasm or endovascular treatment was necessary.

\section{Statistical analysis}

SPSS 17.0 (SPSS Inc, Chicago, Illinois) was used for performing statistical analyses and $P<0.05$ was considered significant. For continuous variables, they were shown as mean \pm standard deviation, and means were analyzed by the 2-sample $t$ test. For categorical variables, they were expressed as counts (percentage), and the Pearson $X^{2}$ test or Fisher exact test was used for their analysis. To identify predictors of SCV, multivariate logistic regression model analysis was performed. Briefly, all available variables, including those on demographic, prior medical history, admission, and aneurysm surgical variables that had univariate associations $P<0.25$ with the outcome variable $(\mathrm{SCV})$ were included in the initial pool of potential predictors. In order to remove least non-significant variables, backward stepwise regression was performed. These variables were removed one by one, until all the remaining potential predictors had $P<0.05$. Area under the curve (AUC) model performance was calculated using the $Z$ test. In this model, AUC ranges from 0.5 to 1.0 , indicating predictive power from weak to strong. In order to calculate the corresponding sensitivities and specificities of the variables, the best thresholds for WFNS grades, modified Fisher grades, and Lp-PLA2 levels on admission were used. They were derived from the receiver operating characteristic (ROC) curve analyses. Using the Kaplan-Meier method, the percentage of patients surviving SCV for 30 days was calculated. After the survival curves were drawn, the log-rank test was used to compare them.

\section{Results}

\section{Patient characteristics}

A total of 128 patients were enrolled in this study and categorized into SCV and non-SCV groups. The age range of patients was $53.44 \pm 10.51$ years, with $70 / 128$ being female. Patient demographics, prior medical history, clinical characteristics, medical complications, and admission Lp-PLA2 level were compared, and results are shown in Table 1. The average admission Lp-PLA2 level of patients with SCV was significantly higher than that of patients without SCV (Table 1).

\section{Lp-PLA2 at admission for SCV patients}

For this cohort of patients, 12 (9.4\%) experienced SCV within 21 days after aSAH. Both univariate and multivariate models were used to analyze variables that might be associated with SCV. These variables included WFNS grade, modified Fisher grade, diabetes mellitus, aneurysm size, and Lp-PLA2 level. The results showed that both the modified Fisher grade $(\mathrm{OR}=10.08,95 \% \mathrm{CI}=2.04-49.86$, $P=0.005)$ and the Lp-PLA2 level $(\mathrm{OR}=6.66,95 \% \mathrm{CI}=$ 1.33-33.30, $P=0.021)$ were predictors that are significantly associated with SCV (Table 2).

The Lp-PLA2 level for assessing the predictive performance of SCV is represented as AUC $=0.696$ (95\% $\mathrm{CI}=0.536-0.856)$, as revealed by ROC curve analysis. Using the best threshold of $169.3 \mu \mathrm{g} / \mathrm{L}$ for Lp-PLA2, the sensitivity was derived as $83.3 \%$ and the specificity was derived as $51.7 \%$ (Fig. 1). The predictive performance of the Lp-PLA2 was similar to that of WFNS grade (AUC $=0.726,95 \% \mathrm{CI}=0.591-0.862 ; Z=0.280, P=0.780)$ and modified Fisher grade (AUC $=0.778,95 \% \mathrm{CI}=$ 0.637-0.920; $Z=0.751, P=0.452$ ) in aSAH (Fig. 1). 
Table 1 Patient characteristics by occurrence of symptomatic cerebral vasospasm

\begin{tabular}{|c|c|c|c|c|}
\hline Characteristics & Total $(n=128)$ & $\operatorname{SCV}(n=12)$ & No SCV $(n=116)$ & $P$ value \\
\hline \multicolumn{5}{|l|}{ Demographics } \\
\hline Age, year & $53.44 \pm 10.51$ & $55.00 \pm 9.95$ & $53.28 \pm 10.59$ & 0.590 \\
\hline Gender, female & 70 (54.69\%) & 7 (58.33\%) & $63(54.31 \%)$ & 0.790 \\
\hline \multicolumn{5}{|l|}{ Admission clinical grade } \\
\hline WFNS grade & & & & 0.007 \\
\hline Grade I & $56(43.75 \%)$ & $1(8.33 \%)$ & 55 (47.41\%) & \\
\hline Grade ॥ & $6(4.69 \%)$ & $1(8.33 \%)$ & $5(4.31 \%)$ & \\
\hline Grade III & $14(10.94 \%)$ & $2(16.67 \%)$ & $12(10.34 \%)$ & \\
\hline Grade IV & $30(23.44 \%)$ & $3(25.00 \%)$ & $27(23.28 \%)$ & \\
\hline Grade V & $22(17.19 \%)$ & $5(41.67 \%)$ & $17(14.66 \%)$ & \\
\hline \multicolumn{5}{|l|}{ Admission blood pressure } \\
\hline $\mathrm{SAP}, \mathrm{mmHg}$ & $146.36 \pm 23.97$ & $140.08 \pm 25.50$ & $147.01 \pm 23.83$ & 0.343 \\
\hline $\mathrm{DAP}, \mathrm{mmHg}$ & $83.88 \pm 11.70$ & $81.42 \pm 9.31$ & $84.14 \pm 11.92$ & 0.445 \\
\hline MAP, mmHg & $104.71 \pm 14.34$ & $100.97 \pm 13.99$ & $105.09 \pm 14.38$ & 0.345 \\
\hline \multicolumn{5}{|l|}{ Admission $C T$ scan grade } \\
\hline Modified Fisher grade & $2(2-3)$ & $2(2-3)$ & $4(3-4)$ & $<0.001$ \\
\hline \multicolumn{5}{|l|}{ Medical history } \\
\hline Hypertension & $57(44.53 \%)$ & 7 (58.33\%) & $50(43.10 \%)$ & 0.312 \\
\hline Diabetes mellitus & $16(12.50 \%)$ & $3(25.00 \%)$ & $13(11.21 \%)$ & 0.174 \\
\hline Cardiovascular disease & $19(14.84 \%)$ & $3(25.00 \%)$ & $16(13.79 \%)$ & 0.385 \\
\hline Smoking history & $35(27.34 \%)$ & $4(33.33 \%)$ & $31(26.72 \%)$ & 0.735 \\
\hline \multicolumn{5}{|l|}{ Aneurysm characteristics } \\
\hline Aneurysm size, mm & $7.25 \pm 4.24$ & $8.75 \pm 6.22$ & $7.09 \pm 3.99$ & 0.197 \\
\hline Multiple aneurysms & $35(27.34 \%)$ & $3(25.00 \%)$ & $32(27.59 \%)$ & 1.000 \\
\hline Anterior circulation & 94 (73.44\%) & $9(75.00 \%)$ & $85(73.28 \%)$ & 1.000 \\
\hline Clipping & $109(85.16 \%)$ & $11(91.67 \%)$ & $98(84.48 \%)$ & 1.000 \\
\hline \multicolumn{5}{|l|}{ Laboratory } \\
\hline$L p-P L A 2, \mu g / L$ & $182.09 \pm 60.09$ & $224.47 \pm 76.14$ & $177.70 \pm 56.79$ & 0.010 \\
\hline \multicolumn{5}{|l|}{ Medical complications } \\
\hline Pneumonia & $51(39.84 \%)$ & $8(66.67 \%)$ & $43(37.07 \%)$ & 0.063 \\
\hline Intracranial infection & $11(8.59 \%)$ & $1(8.33 \%)$ & $10(8.62 \%)$ & 1.000 \\
\hline Sepsis & $5(3.91 \%)$ & $1(8.33 \%)$ & $4(3.45 \%)$ & 0.394 \\
\hline Hydrocephalus & $18(14.06 \%)$ & $2(16.67 \%)$ & $16(13.79 \%)$ & 0.677 \\
\hline
\end{tabular}

Values are $n(\%)$, mean \pm SD, median $(25-75 \%)$

$S C V$ symptomatic cerebral vasospasm, SAP systolic arterial pressure, DAP diastolic arterial pressure, MAP mean arterial pressure, $L p-P L A 2$ lipoprotein-associated phospholipase A2

The non-SCV rate analysis of patients with different WFNS grades with and without elevated Lp-PLA2

ROC curve analysis showed that the predictive performances of the Lp-PLA2 and WFNS grade were similar. We further analyzed the relationship between the LpPLA2 and the occurrence of SCV in patients with different WFNS grades. The Kaplan-Meier curve shows a 30 days non-SCV rate of 75\% (21/28) for poor WFNS grade patients with poor Lp-PLA2 level (> $200 \mu \mathrm{g} / \mathrm{L}$ ), of $92.1 \%$ $(35 / 38)$ for poor WFNS grade patients with good Lp-
PLA2 level $(\leq 200 \mu \mathrm{g} / \mathrm{L})$, of $90.0 \%$ (9/10) for good WFNS grade patients (admission WFNS grade $<3$ ) with poor Lp-PLA2 level, and of 98.1\% (51/52) for good WFNS grade patients with good Lp-PLA2 level. $P=0.006$ as calculated in the log-rank test. Post hoc log-rank testing revealed that in the good WFNS grade patient subgroup, patients with poor Lp-PLA2 level had a similar non-SCV rate to that of patients with good Lp-PLA2 level $(P=0.196)$; but in the poor WFNS grade patient sub-group, patients with poor Lp-PLA2 level had 
Table 2 Multivariate model analysis of SCV with admission predictors

\begin{tabular}{|c|c|c|c|c|c|c|}
\hline \multirow[t]{2}{*}{ Predictors* } & \multicolumn{4}{|c|}{ Univariate analysis } & \multicolumn{2}{|c|}{ Multivariate analysis $^{\ddagger}$} \\
\hline & $\mathrm{SCV}(n=12)$ & No SCV $(n=116)$ & OR $(95 \% \mathrm{Cl})$ & $P$ value & OR $(95 \% \mathrm{Cl})$ & $P$ value \\
\hline WFNS grade $\geq 2^{\dagger}$ & $11(91.67 \%)$ & $61(52.59 \%)$ & $9.92(1.24,79.33)$ & 0.031 & & \\
\hline Modified Fisher grade $\geq 3^{+}$ & $10(83.33 \%)$ & $43(37.07 \%)$ & $8.49(1.78,40.57)$ & 0.007 & $10.08(2.04,49.86)$ & 0.005 \\
\hline Diabetes mellitus & $3(25.00 \%)$ & $13(11.21 \%)$ & $2.64(0.63,11.02)$ & 0.183 & & \\
\hline Aneurysm size $\geq 7.5 \mathrm{~mm}^{\dagger}$ & $4(33.33 \%)$ & $46(39.66 \%)$ & $0.76(0.22,2.67)$ & 0.670 & & \\
\hline Lp-PLA2 > $169.3 \mu \mathrm{g} / \mathrm{L}^{\dagger}$ & $10(83.33 \%)$ & $56(48.28 \%)$ & $5.36(1.12,25.53)$ & 0.035 & $6.66(1.33,33.30)$ & 0.021 \\
\hline
\end{tabular}

*Predictors include all admission variables in Table 1 that have $P<0.25$

${ }^{\dagger}$ The cut-off point was calculated on the basis of ROC curve analysis

${ }^{\ddagger}$ All variables having $P<0.05$ from univariate analysis were included in multivariate analysis. Backward stepwise regression methods were performed to create the final model, whereby the least nonsignificant variable was removed from the model one at a time, until all remaining variables had $P<0.05$

significantly lower non-SCV rate than that of patients having good Lp-PLA2 level $(P=0.045)$ (Fig. 2).

\section{Discussion}

The current study demonstrates that the average LpPLA2 level of patients with SCV was significantly higher than that of patients without SCV. Furthermore, in multivariate logistic regression models of finding predictors of SCV, Lp-PLA2 level was an independent and significant predictor after adjustment for confounders, including the WFNS grade, diabetes mellitus, and aneurysm size. Currently, among all grading schemes, the modified Fisher grade and the WFNS grade are the most widely used, because of their relatively strong predictive power and ease of use [4-6]. It was found that for SCV patients, the predictive performance of LpPLA2 was comparable with the WFNS grade and modified Fisher grade, which indicates the potential of the Lp-PLA2 level as a new marker in aSAH prognosis.

As one of the worst and most common complications of aSAH, CV usually occurs within 1 month post-aSAH and is associated with unfavorable outcome [26]. Despite improvements in early CT diagnosis and neurosurgical critical care, approximately $30-70 \%$ of aSAH patients will experience ACV, approximately $10-30 \%$ of aSAH patients will experience SCV, and nearly $15 \%$ patients

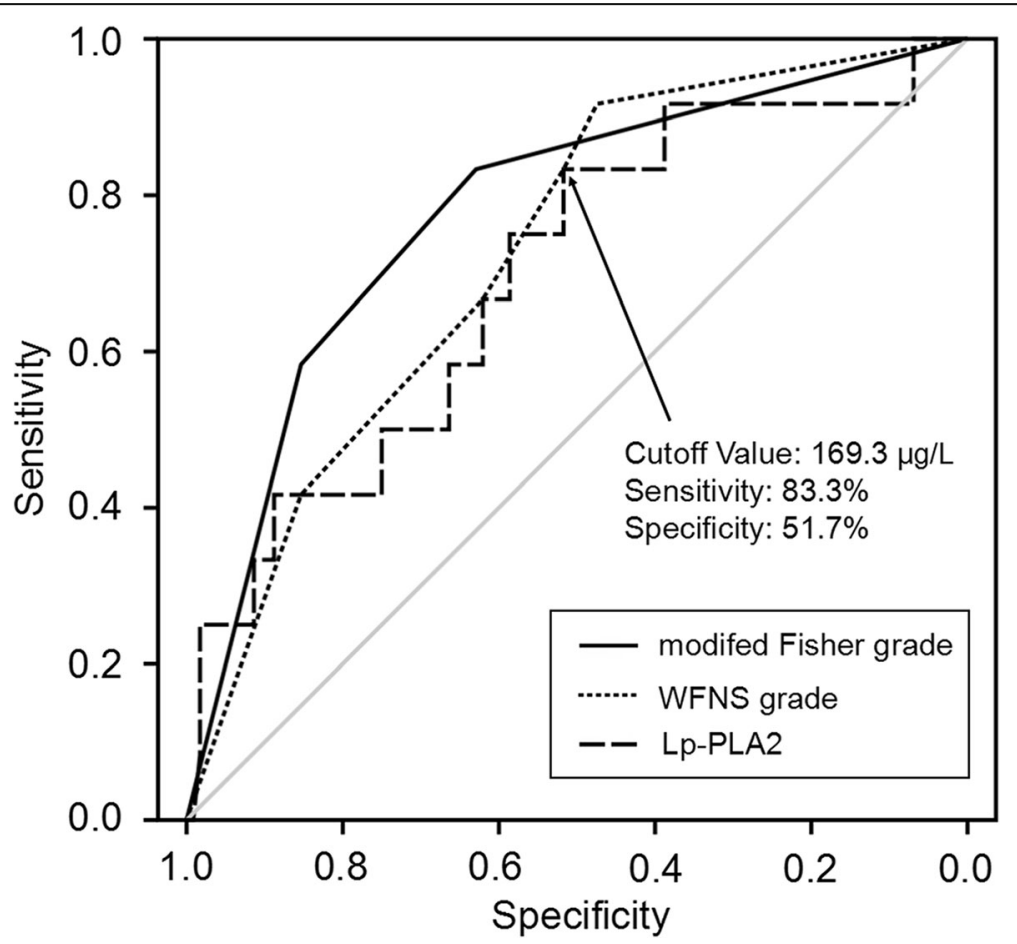

Fig. 1 Comparisons of AUC for identifying SCV using LP-PLA2, the WFNS grade, and modified Fisher grade. ROC curves were constructed on the basis of the sensitivity and specificity of the WFNS grade, modified Fisher grade, and Lp-PLA2 for identifying SCV. Z test was used for comparing AUC performances and results revealed that the predictive performance of the Lp-PLA2 was similar to that of the WFNS grade $(Z=0.280, P=$ $0.780)$ and modified Fisher grade $(Z=0.751, P=0.452)$. 


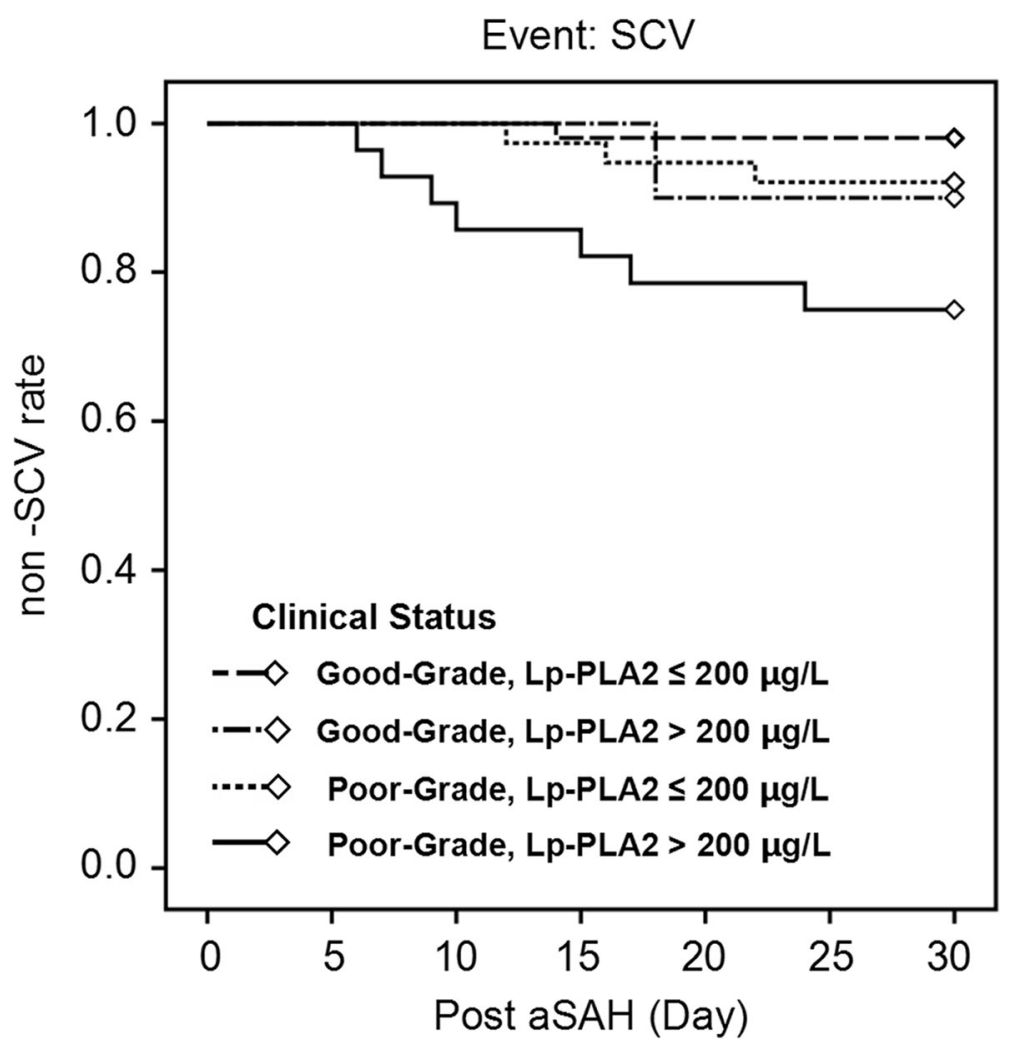

Fig. 2 The Kaplan-Meier curve of the 30 days non-SCV rate of patients with and without elevated admission Lp-PLA2. Post hoc log-rank testing revealed that the good WFNS grade patients having poor Lp-PLA2 level $(>200 \mu \mathrm{g} / \mathrm{L})$ had a similar non-SCV rate to that of good WFNS grade patients having good Lp-PLA2 level $(\leq 200 \mu \mathrm{g} / \mathrm{L})(P=0.712)$, but the poor WFNS grade patients having poor Lp-PLA2 level had significantly lower non-SCV rate than poor WFNS grade patients having good Lp-PLA2 level $(P=0.001)$

will die or have devastating neurologic outcomes [20, 27, 28]. Currently, the pathogenesis and etiology of CV are still poorly understood. Although there are a wide variety of treatments to prevent post-aSAH CV, their effectiveness differs significantly. The treatment of $\mathrm{CV}$ via medication is usually suboptimal and often associated with cerebral ischemia or infraction, further worsening the condition of patient. Therefore, if early prognosis of $\mathrm{CV}$ could be achieved to predict potential risks, optimized care and more effective distribution of healthcare resources might be made possible for these patients. There have been studies reporting on the connection between elevated Lp-PLA2 level and ACV in aSAH patients [19]. The associated morbidity and mortality of SCV could be higher than ACV. However, whether LpPLA2 can predict the occurrence of SCV in aSAH patients is still unclear.

Based on published literature, the possible causes of post-aSAH CV include endothelial cell interactions, alterations in calcium signaling, oxidative processes, spreading depolarization, and/or inflammation [29, 30]. For goal-oriented therapy, the aim is to create an optimal physiological environment for the comatose injured brain, and technologies such as detection of related biomarkers may eventually be used. Biomarker measurements, usually quantitative, can be utilized in diagnosis and monitoring of patient response to treatment. Although there exist established biomarkers of predicting aSAH, there is a need for better use of them and more reliable ones, so a more effectively guided clinical diagnosis and assessment of aSAH can be achieved [12-14]. Biomarkers such as $\mathrm{C}$-reactive protein, which are related to inflammatory reaction, can be used to predict $\mathrm{CV}$ after aSAH [31, 32]. Compared to C-reactive protein, which has high-sensitivity, LP-PLA2 could be more specific when predicting inflammation in vessels [33]. More and more animal and human studies have been done to explore clinical application of Lp-PLA2. Results showed that the plasma LP-PLA2 level is positively related to oxidative stress, atherosclerosis, and various cerebrovascular and cardiovascular events including ischemic and hemorrhagic strokes [17, 18, 34].

The rate of SCV was $9.4 \%$ in our cohort of patients. The levels of the LP-PLA2 within the first $24 \mathrm{~h}$ were significantly higher in our cohort of aSAH patients who experienced SCV compared to those who did not. In the 
multivariate logistic regression model analysis, LP-PLA2 was an independent and significant predictor of SCV. Based on past research [25], poor Lp-PLA2 level indicates moderate inflammation of the vessels and an increased risk of cardiovascular disease. In this study, the best thresholds of Lp-PLA2 level used in predicting SCV were lower than $200 \mu \mathrm{g} / \mathrm{L}$. Using lower Lp-PLA2 level to predict SCV improved sensitivity but decreased specificity. However, considering the severity of SCV, predicting SCV using biomarkers with high sensitivity might be helpful for identifying more patients at high risk of SCV. Using the best threshold of $169.3 \mu \mathrm{g} / \mathrm{L}$, the specificity of Lp-PLA2 level for predicting SCV was calculated to be $51.7 \%$, and the sensitivity was $83.3 \%$, which is a relatively high for diagnostic purpose (> 80\%).

The clinical grading schemes for assessing severity of aSAH and interpretation of CT scans, such as the WFNS grade and modified Fisher grade [4-6], are often used to predict occurrence of CV. However, identification of more easily measurable biomarkers for predicting $\mathrm{CV}$ would still be helpful for prognosis and risk mitigation. Lp-PLA2 is secreted by endothelium inflammatory cells and then released into the blood, and it is a specific marker that reflects degree of inflammation of the vessel endothelium. It can be easily obtained and integrated into daily practice. Compared to subjective clinical grades, Lp-PLA2 might be more objective and might be able to distinguish changes in the physical condition of a patient that may be missed when using clinical grades, which could lead to modifications in the treatment plan. In our study, the ROC curve analysis revealed that the predictive performance of the Lp-PLA2 was comparable to that of WFNS grade. Therefore, we further analyzed the relationship between Lp-PLA2 and the occurrence of SCV in patients with different WFNS grades. It was found that the elevated admission LP-PLA2 level may have clinical value especially for patients with poor WFNS grade (WFNS grade $\geq 3$ ). For good WFNS grade patients, those with poor Lp-PLA2 level $(>200 \mu \mathrm{g} / \mathrm{L})$ and with good Lp-PLA2 level $(\leq 200 \mu \mathrm{g} / \mathrm{L})$ had similar non-SCV rate; but for poor WFNS grade patients, those with poor Lp-PLA2 level had significantly lower nonSCV rate than patients with good Lp-PLA2 level $(P=$ $0.045)$. This result suggests that Lp-PLA2 might be used in combination with WFNS in predicting the SCV. These patients with poor WFNS grade and admission Lp-PLA2 level > $200 \mu \mathrm{g} / \mathrm{L}$ might need more careful post-surgery monitoring.

There are still a number of limitations of this study, including its observational design. First, ELISA, the most common method of detecting level of Lp-PLA2 was used in our study. However, this level might change when a different measurement method is used [35]. Therefore, one of the key issues needs to be addressed is the standardization of Lp-PLA2 measurement method. Second, not many SCV cases were included in this study, and more data with higher number of enrolled patients are still needed to confirm findings in this study.

\section{Conclusions}

In this study, it was found that the Lp-PLA2 level obtained within the first $24 \mathrm{~h}$ post-aSAH was associated with SCV following aSAH. The Lp-PLA2 might be helpful as a marker easily obtained in the clinical setting for predicting SCV in patients with aSAH. In order to determine if changes in Lp-PLA2 over time and the onset of $\mathrm{SCV}$ are related to each other, further research is still needed.

\section{Abbreviations}

aSAH: Aneurismal subarachnoid hemorrhage; Lp-PLA2: Lipoproteinassociated phospholipase A2; SCV: Symptomatic cerebral vasospasm; WFNS: World Federation of Neurosurgical Societies; ACV: Angiographic cerebral vasospasm; CTA: Computed tomography angiography; DSA: Digital subtraction angiography; GCS: Glasgow coma scale; ELISA: Enzyme-linked immunosorbent assay; COV: Coefficient of variation; TOF: Time-of-flight; AUC: Under the curve; ROC: Receiver operating characteristic

\section{Acknowledgements}

This study was supported by grants from the key clinical specialty discipline construction program of Fujian, P.R.C. and the major project of Fujian Provincial Department of Science and Technology (no. 2014YZ0003).

\section{Authors' contributions}

C.Y.D. and F.Y.W. were major contributors in writing the manuscript. H.P.C., X.Y.C., S.F.Z., L.H.Y., Y.X.L., and Z.Y.L. analyzed and interpreted the patient data. D.Z.K. designed the study. All authors read and approved the final manuscript.

\section{Funding}

This study was supported by grants from the key clinical specialty discipline construction program of Fujian, P.R.C. and the major project of Fujian Provincial Department of Science and Technology (no. 2014YZ0003). The funding body played an important role in the design of the study.

Availability of data and materials

All data generated or analyzed during this study are included in the article.

\section{Ethics approval and consent to participate}

The study was approved by the Ethics Committee of the First Affiliated Hospital of Fujian Medical University (Fujian, China). Committee's reference number: [2014]059. Informed consent was obtained from the patients or their authorized legal representative if patients cannot sign the form themselves.

\section{Consent for publication}

Not applicable

\section{Competing interests}

The authors declare that they have no competing interests in this section.

Received: 12 September 2019 Accepted: 9 March 2020

Published online: 04 April 2020

\section{References}

1. Mijiti M, Mijiti P, Axier A, et al. Incidence and predictors of angiographic vasospasm, symptomatic vasospasm and cerebral infarction in Chinese patients with aneurysmal subarachnoid hemorrhage. PLoS One. 2016;11(12): e0168657.

2. Konczalla J, Kashefiolasl S, Brawanski N, et al. Cerebral vasospasmdependent and cerebral vasospasm-independent cerebral infarctions 
predict outcome after nonaneurysmal subarachnoid hemorrhage: a singlecenter series with 250 patients. World Neurosurg. 2017;106:861-9 e864.

3. Findlay JM, Nisar J, Darsaut T. Cerebral vasospasm: a review. Can J Neurol Sci. 2016;43(1):15-32.

4. Lee Y, Zuckerman SL, Mocco J. Current controversies in the prediction, diagnosis, and management of cerebral vasospasm: where do we stand? Neurol Res Int. 2013;2013:373458.

5. Dupont SA, Wijdicks EF, Manno EM, Lanzino G, Rabinstein AA. Prediction of angiographic vasospasm after aneurysmal subarachnoid hemorrhage: value of the Hijdra sum scoring system. Neurocrit Care. 2009;11(2):172-6.

6. Dilvesi $D$, Cigic $T$, Papic $V$, et al. The Fisher grade in predicting a degree of cerebral vasospasm in patients after intracranial aneurysm rupture. Vojnosanit Pregl. 2016;73(4):349-52.

7. Wilson DA, Nakaji P, Abla AA, et al. A simple and quantitative method to predict symptomatic vasospasm after subarachnoid hemorrhage based on computed tomography: beyond the Fisher scale. Neurosurgery. 2012;71(4): 869-75.

8. Kramer AH, Hehir M, Nathan B, et al. A comparison of 3 radiographic scales for the prediction of delayed ischemia and prognosis following subarachnoid hemorrhage. J Neurosurg. 2008;109(2):199-207.

9. Klimo P Jr, Schmidt RH. Computed tomography grading schemes used to predict cerebral vasospasm after aneurysmal subarachnoid hemorrhage: a historical review. Neurosurg Focus. 2006;21(3):E5.

10. Friedman JA, Goerss SJ, Meyer FB, et al. Volumetric quantification of Fisher Grade 3 aneurysmal subarachnoid hemorrhage: a novel method to predict symptomatic vasospasm on admission computerized tomography scans. J Neurosurg. 2002;97(2):401-7.

11. Smith ML, Abrahams JM, Chandela S, et al. Subarachnoid hemorrhage on computed tomography scanning and the development of cerebral vasospasm: the Fisher grade revisited. Surg Neurol. 2005;63(3):229-34 discussion 234-225.

12. Carpenter KL, Czosnyka M, Jalloh I, et al. Systemic, local, and imaging biomarkers of brain injury: more needed, and better use of those already established? Front Neurol. 2015;6:26.

13. Abboud T, Mende KC, Jung R, et al. Prognostic value of early $\mathrm{S} 100$ calcium binding protein $B$ and neuron-specific enolase in patients with poor-grade aneurysmal subarachnoid hemorrhage: a pilot study. World Neurosurg. 2017; 108:669-75.

14. Nakatsuka Y, Shiba M, Nishikawa H, et al. Acute-phase plasma osteopontin as an independent predictor for poor outcome after aneurysmal subarachnoid hemorrhage. Mol Neurobiol. 2018;55(8):6841-9.

15. Tian Y, Jia H, Li S, et al. The associations of stroke, transient ischemic attack, and/or stroke-related recurrent vascular events with Lipoprotein-associated phospholipase A2: a systematic review and meta-analysis. Medicine (Baltimore). 2017;96(51):e9413.

16. Wang Y, Zhang J, Qian Y, et al. Association of Lp-PLA2 mass and aysmptomatic intracranial and extracranial arterial stenosis in hypertension patients. PLoS One. 2015;10(6):e0130473.

17. Li D, Wei W, Ran X, et al. Lipoprotein-associated phospholipase A2 and risks of coronary heart disease and ischemic stroke in the general population: a systematic review and meta-analysis. Clin Chim Acta. 2017;471:38-45.

18. Rosso C, Rosenbaum D, Pires C, et al. Lipoprotein-associated phospholipase A2 during the hyperacute stage of ischemic and hemorrhagic strokes. J Stroke Cerebrovasc Dis. 2014;23(4):e277-82.

19. Ding CY, Cai HP, Ge HL, et al. Is admission lipoprotein-associated phospholipase A2 a novel predictor of vasospasm and outcome in patients with aneurysmal subarachnoid hemorrhage? Neurosurgery. 2019; pii: nyz041. doi: https://doi.org/10.1093/neuros/nyz041.

20. Inagawa T. Risk factors for cerebral vasospasm following aneurysmal subarachnoid hemorrhage: a review of the literature. World Neurosurg. 2016; 85:56-76.

21. Kanamaru K, Suzuki H, Taki W. Cerebral infarction after aneurysmal subarachnoid hemorrhage. Acta Neurochir Suppl. 2016;121:167-72.

22. Cai H, Zheng $\mathrm{S}$, Cai B, et al. Neuroglobin as a novel biomarker for predicting poor outcomes in aneurysmal subarachnoid hemorrhage. World Neurosurg. 2018;116:e258-65

23. Connolly ES Jr, Rabinstein AA, Carhuapoma JR, et al. Guidelines for the management of aneurysmal subarachnoid hemorrhage: a guideline for healthcare professionals from the American Heart Association/American Stroke Association. Stroke. 2012;43(6):1711-37.
24. Diringer MN, Bleck TP, Claude Hemphill J 3rd, et al. Critical care management of patients following aneurysmal subarachnoid hemorrhage: recommendations from the Neurocritical Care Society's Multidisciplinary Consensus Conference. Neurocrit Care. 2011:15(2):211-40.

25. Tsai AK, Steffen BT, Ordovas JM, et al. Short-term fenofibrate treatment reduces elevated plasma Lp-PLA2 mass and SVCAM-1 levels in a subcohort of hypertriglyceridemic GOLDN participants. Transl Res. 2011;158(2):99-105.

26. Wilkins RH. Cerebral vasospasm. Crit Rev Neurobiol. 1990;6(1):51-77.

27. Przybycien-Szymanska MM, Ashley WW Jr. Biomarker discovery in cerebral vasospasm after aneurysmal subarachnoid hemorrhage. J Stroke Cerebrovasc Dis. 2015;24(7):1453-64.

28. Skoch J, Tahir R, Abruzzo T, et al. Predicting symptomatic cerebral vasospasm after aneurysmal subarachnoid hemorrhage with an artificial neural network in a pediatric population. Childs Nerv Syst. 2017;33(12):2153-7.

29. Haruma J, Teshigawara K, Hishikawa T, et al. Anti-high mobility group box-1 (HMGB1) antibody attenuates delayed cerebral vasospasm and brain injury after subarachnoid hemorrhage in rats. Sci Rep. 2016;6:37755.

30. Lin CL, Dumont AS, Zhang JH, Zuccarello M, Muroi C. Cerebral vasospasm after aneurysmal subarachnoid hemorrhage: mechanism and therapies. Biomed Res Int. 2014;2014:679014.

31. Fountas KN, Tasiou A, Kapsalaki EZ, et al. Serum and cerebrospinal fluid Creactive protein levels as predictors of vasospasm in aneurysmal subarachnoid hemorrhage. Clinical article. Neurosurg Focus. 2009;26(5):E22.

32. Jeon $\mathrm{YT}$, Lee JH, Lee $\mathrm{H}$, et al. The postoperative C-reactive protein level can be a useful prognostic factor for poor outcome and symptomatic vasospasm in patients with aneurysmal subarachnoid hemorrhage. J Neurosurg Anesthesiol. 2012;24(4):317-24.

33. Dallmeier D, Koenig W. Strategies for vascular disease prevention: the role of lipids and related markers including apolipoproteins, low-density lipoproteins (LDL)-particle size, high sensitivity C-reactive protein (hs-CRP), lipoprotein-associated phospholipase A2 (Lp-PLA(2)) and lipoprotein(a) (Lp(a)). Best Pract Res Clin Endocrinol Metab. 2014;28(3):281-94.

34. Lin J, Zheng H, Cucchiara BL, et al. Association of Lp-PLA2-A and early recurrence of vascular events after TIA and minor stroke. Neurology. 2015; 85(18):1585-91.

35. Topbas C, Swick A, Razavi M, et al. Measurement of lipoprotein-associated phospholipase A2 by use of 3 different methods: exploration of discordance between ELISA and activity assays. Clin Chem. 2018;64(4):697-704.

\section{Ready to submit your research? Choose BMC and benefit from:}

- fast, convenient online submission

- thorough peer review by experienced researchers in your field

- rapid publication on acceptance

- support for research data, including large and complex data types

- gold Open Access which fosters wider collaboration and increased citations

- maximum visibility for your research: over $100 \mathrm{M}$ website views per year

At BMC, research is always in progress.

Learn more biomedcentral.com/submissions 